\title{
A Cross Sectional Study to assess prevalence of Computer Vision Syndrome and vision related problems in Computer Users
}

\author{
Authors \\ Dr (Prof) M K Rathore ${ }^{1}$, Dr Vinanti Kangale ${ }^{2}$, Dr (Prof) C V Kulkarni ${ }^{3}$ \\ Dr Preeti Rawat ${ }^{4}$, Dr Shweta Walia ${ }^{5}$ \\ ${ }^{1}$ Professor Dept of Ophthalmology, MGM Medical College Indore \\ Email- mkrathore@hotmail.com \\ ${ }^{2}$ PG Resident Dept of Ophthalmology, MGM Medical College Indore \\ Email-vinantikangale.vk@gmail.com \\ ${ }^{3}$ Professor and Head Dept Of Pathology MGM Medical College Indore \\ Email:dr_chandrahaskulkarni@yahoo.com \\ ${ }^{4}$ Associate professor Dept of Ophthalmology, MGM Medical College Indore \\ Email:drpreeti.eye@rediffmail.com \\ ${ }^{5}$ Assistant professor Dept of Ophthalmology, MGM Medical College Indore \\ Email:drshweta.2007@gmail.com
}

\begin{abstract}
The aim was to assess prevalence of computer vision syndrome and find association of various factors with occurrence of symptoms.

Methods- In this cross-sectional study participant were surveyed using self administered questionnaire and underwent complete ophthalmological and dry eye assessment.

Results- $A$ total of 150 participants aged 18-40 years were examined. Majority of the patients were in the age group of 26-30 years, who accounted for 57 cases i.e. 38\% of total cases. Male and Female sex distribution was unequal in this study. Males 81 (54\%) and females 69 (46\%). The prevalence of symptoms of CVS was found to be $75.33 \%$ (113/150); most disturbing symptom was headache 101/150 (67.33\%), tired eyes 76(50.66\%), pain behind eyes 74(49.33\%) Majority of patients 97 (64.66\%) used computer for 4-6 hours. Abnormal TBUT 56 (37.4\%), Schirmer's Test II 42 (28\%), Tear height meniscus 59 (39.4\%), fluroscein staining findings 15 (10\%), lissamine green staining findings $20(13.33 \%)$ and conjunctival impression cytology findings were reported as mild dysplasia in 25 (16.66\%), moderate dysplasia 7(4.66\%), severe dysplasia 3 (2\%), acute inflammatory lesions 5 (3.33\%) \& inclusion of Chlamydia trachomatis body was seen in 1. Knowledge about CVS was present in 65(43.33\%).
\end{abstract}

Conclusion- Most participants had CVS and its severity was correlated with prolonged working hours on computer. There was marked unawareness about CVS.

Keywords- Computer vision syndrome, conjunctival impression cytology, fluroscein staining, lissamine green staining, tear film break up time, Schirmer's test. 


\section{INTRODUCTION}

Computers are being used increasingly by large number of people today. There has been a great advancement in the information technology in the past few decades. The use of computer has made life easier and has increased the work productivity tremendously ${ }^{[1]}$. In this present era, with excessive computer usage there has been an outbreak in computer related health problems. This has led to an increase in the number of patients complaining about ocular and non ocular symptoms related to computer use which are being grouped together as COMPUTER VISION SYNDROME (CVS).

Computer has become an integral part of office equipments and in routine life. However, working at a computer terminal is not free from health hazards and computer users are experiencing a variety a vision related complaints which is collectively referred to as Computer Vision Syndrome(CVS) (Tamez-Gonzalez et al, 2003) $^{[2] .}$

American Optometric Association (AOA) has defined Computer Vision Syndrome as a complex of eye and vision associated problems mainly related to activities which stresses the near vision and which are experienced in relation or during the use of computer (Americian optometric Association 2010, Loh and Reddy, 2008). ${ }^{[1][3]}$

Blehm et al, 2005 ${ }^{[4]}$ categorized the symptoms in four major categories:

i. Asthenopic-eye strain, tired eyes, sore eyes

ii. Ocular Surface Related-watering, irritation, dry eye

iii. Visual-blurred vision, slowness of focus change, double vision and

iv. Extraocular-neck pain, backache, shoulder pain

Numerous factors accounting for Computer Vision Syndrome are duration of usage, poor lighting, screen brightness, environmental factors, vision problems \& improper work station set up also account for eye \& visual problems associated with computer.

The aim of this study is to identify the prevalence of CVS AND major visual and systemic manifestations among the computer users also enlighten the public health professionals on the need of spreading awareness regarding health hazards associated with computer use.

\section{MATERIALS AND METHODS}

Study was cross sectional study conducted in MGM Medical college, Indore between 2015 July-2015 june. The study was approved by the local ethics committee and written informed consent was obtained from all subjects prior to participation.

All participants were surveyed using self administered questionnaire and complete ophthalmic examination was done.

A total of 150 computer users below 40 years age were included.

\section{Inclusion Criteria}

1. Subject working on computer for atleast 3 hours per day daily since 6 months and not using lubricating drops

2. Subject giving consent for the study

\section{Exclusion Criteria}

1. Subject who did not give consent for the study

2. Subjects older than 40 years are excluded because of age induced vision problems of presbyopia

3. Contact lens users

4. Known case of dry eye disease and ocular motility disorder.

5. Subject using drugs (systemic/topical) causing dry eye

\section{COMPLETE OPHTHALMIC ASSESSMENT}

The patients were examined for their VA on snellen's chart on the first OPD visit. Detailed history (ocular and systemic symptoms) were noted and they were requested to fill the questionnaire provided to them. The detailed examination of patients were done under the following heading:

\section{HISTORY}

a) Subjects were registered with their name, age, sex and address

b) Relevant history from the patients was taken regarding

- Headache, blurring of vision, watering, redness 
- Shoulder pain, neck pain, backache

- Rule out conditions associated with dry eye

\section{EXAMINATION}

a) A comprehensive general examination of all subjects was done to rule out any systemic illness.

Any history regarding diabetes, hypertension, Rheumatoid Arthritis, Alcohol abuse, smoking, tobacco intake was be noted.

\section{b) ON OCULAR EXAMINATION}

1) Vision TestingBCVA DISTANT VISION-

Right eye-

Left eye-

BCVA NEAR VISION

Right eye-

Lefteye-.

2) SLIT LAMP EXAMINATION-

A) Detailed Anterior segment examination under diffuse illumination-

B) Posterior segment examination-

3) TEAR FILM ANALYSIS:

i) Tear Film Meniscus Height-

ii) TBUT TEST-

iii) Fluroscein Staining Findings-

iv) Lissamine green staining findings-

v) Schirmer's Test Findings-

4) Conjunctival Impression Cytology

\section{RESULTS}

Table 1- Distribution of study participants as per gender

\begin{tabular}{|l|l|l|l|l|l|l|}
\hline \multirow{2}{*}{ Gender } & \multicolumn{2}{|l|}{$\begin{array}{l}\text { Number } \\
\text { Cases }\end{array}$} & \multicolumn{2}{l|}{$\begin{array}{l}\text { CVS } \\
\text { Symptoms } \\
\text { Present }\end{array}$} & \multicolumn{3}{l|}{$\begin{array}{l}\text { No } \\
\text { Symptoms }\end{array}$} \\
\cline { 2 - 7 } & Total & $\%$ & Total & $\%$ & Total & $\%$ \\
\hline Male & 81 & 54 & 59 & 72.83 & 22 & 27.17 \\
\hline Female & 69 & 46 & 54 & 78.26 & 15 & 21.74 \\
\hline Total & 150 & 100 & 113 & 75.33 & 37 & 24.67 \\
\hline
\end{tabular}

Table-2 Distribution of Study Participants As Per

Age

\begin{tabular}{|l|l|l|l|l|l|l|}
\hline \multirow{2}{*}{ Age } & \multicolumn{2}{|l|}{$\begin{array}{l}\text { Number Of } \\
\text { Cases }\end{array}$} & $\begin{array}{l}\text { CVS } \\
\text { Symptoms } \\
\text { Present }\end{array}$ \\
\cline { 2 - 7 } \begin{tabular}{l} 
Years \\
\cline { 2 - 7 }
\end{tabular} & 15 & 10 & 11 & 73.4 & \multicolumn{2}{l|}{$\begin{array}{l}\text { No } \\
\text { Symptoms }\end{array}$} \\
\cline { 2 - 7 } & $\%$ & Total & $\%$ & \multicolumn{2}{l|}{ Total } & $\%$ \\
\hline $\begin{array}{l}21-25 \\
\text { Years }\end{array}$ & 52 & 34.66 & 41 & 78.84 & 11 & 21.15 \\
\hline $\begin{array}{l}26-30 \\
\text { Years }\end{array}$ & 61 & 40.66 & 47 & 77.04 & 14 & 22.95 \\
\hline $\begin{array}{l}31-35 \\
\text { Years }\end{array}$ & 15 & 10 & 8 & 53.33 & 7 & 46.66 \\
\hline $\begin{array}{l}36-40 \\
\text { Years }\end{array}$ & 7 & 4.66 & 6 & 86 & 1 & 14 \\
\hline
\end{tabular}

Table-3 Distribution according to Number of Hours Working on Computer per Day

\begin{tabular}{|l|l|l|l|l|l|l|}
\hline \multirow{2}{*}{ Duration } & \multicolumn{2}{|l|}{ Total } & \multicolumn{2}{l|}{ CVS Present } & \multicolumn{2}{l|}{$\begin{array}{l}\text { No } \\
\text { Symptoms }\end{array}$} \\
\cline { 2 - 7 } & Total & $\%$ & Total & $\%$ & Total & $\%$ \\
\hline 3 Hours & 11 & 7.34 & 10 & 90.9 & 1 & 9.09 \\
\hline 4-6 Hours & 97 & 64.66 & 64 & 66.66 & 33 & 34 \\
\hline $7-8$ Hours & 24 & 16 & 22 & 92 & 2 & 8 \\
\hline$>8$ Hours & 18 & 12 & 17 & 94.44 & 1 & 5.5 \\
\hline Total & 150 & 100 & 113 & 75.34 & 37 & 24.66 \\
\hline
\end{tabular}

Table 4:-Distribution of Visual \& Other Complaints

\begin{tabular}{|l|l|l|l|}
\hline \multirow{3}{*}{$\begin{array}{l}\text { Symptom } \\
\text { Category }\end{array}$} & \multirow{2}{*}{ CVS Symptoms } & \multicolumn{2}{|l|}{ Present } \\
\cline { 3 - 4 } & & Total & $\%$ \\
\hline $\begin{array}{l}\text { Ocular } \\
\text { Symptoms }\end{array}$ & Watering eyes & 41 & 27.33 \\
\cline { 2 - 4 } & Dry eyes & 56 & 37.33 \\
\cline { 2 - 4 } & Itchy eyes & 50 & 33.33 \\
\cline { 2 - 4 } & Pain behind eyes & 74 & 49.44 \\
\cline { 2 - 4 } & Tired eyes & 76 & 50.66 \\
\cline { 2 - 4 } & Redness & 57 & 38 \\
\hline Visual symptoms & Blurred vision & 59 & 39.33 \\
& & & \\
\cline { 2 - 4 } & Double vision & 42 & 28 \\
\hline \multirow{5}{*}{$\begin{array}{l}\text { Systemic } \\
\text { symptoms }\end{array}$} & Shoulder pain & 49 & 32.66 \\
\cline { 2 - 4 } & & & \\
\cline { 2 - 4 } & Neck pan & 58 & 38.66 \\
\cline { 2 - 4 } & Back pain & 101 & 67.33 \\
\cline { 2 - 4 } & Headache & 101 & 67.33 \\
\hline
\end{tabular}




\section{JMSCR Vol||04||Issue||06||Page 11007-11012||June}

Table 5: Comparision of TBUT Findings among Computer Users

\begin{tabular}{|c|c|c|c|c|c|c|c|c|c|c|c|c|c|}
\hline \multirow[t]{2}{*}{ Variables } & \multicolumn{2}{|c|}{$\begin{array}{l}\text { Total No. of } \\
\text { Cases }\end{array}$} & \multicolumn{2}{|c|}{$\begin{array}{l}\text { CVS } \\
\text { Symptoms } \\
\text { Present }\end{array}$} & \multicolumn{2}{|c|}{ No Symptoms } & \multirow{2}{*}{$\begin{array}{l}\text { Van } \\
\text { Bijsterveld } \\
\text { Staining }\end{array}$} & \multicolumn{2}{|c|}{$\begin{array}{l}\text { Total No. of } \\
\text { Cases }\end{array}$} & \multicolumn{2}{|c|}{$\begin{array}{l}\text { CVS } \\
\text { Symptoms } \\
\text { Present }\end{array}$} & \multicolumn{2}{|c|}{$\begin{array}{l}\text { No } \\
\text { Symptoms }\end{array}$} \\
\hline & Total & $\%$ & Total & $\%$ & Total & $\%$ & & Total & $\%$ & Total & $\%$ & Total & $\%$ \\
\hline TBUT & 56 & 37.4 & 49 & 88 & 7 & 12 & 0 & 115 & 76.6 & 83 & 72.17 & 32 & 27.83 \\
\hline$(<10 \mathrm{sec})$ & 30 & 37.4 & 49 & 00 & 1 & 12 & $\mathrm{Scc}$ & 12 & 8 & 12 & 100 & 0 & 0 \\
\hline TBUT & 94 & 62.6 & 65 & 69 & 29 & 31 & $\mathrm{Scc}$ & 23 & 15.4 & 20 & 87 & 3 & 13 \\
\hline$(>10 \mathrm{sec})$ & 150 & 02.0 & & & & & Total & 150 & 100 & 115 & 77 & 35 & 23 \\
\hline
\end{tabular}

Table 6:- Distribution of Fluroscein Staining In Computer users

\begin{tabular}{|l|l|l|l|l|l|l|}
\hline $\begin{array}{l}\text { Fluroscein } \\
\begin{array}{l}\text { Staining } \\
\text { In All 5 } \\
\text { Quadrants }\end{array}\end{array}$ & \multicolumn{2}{|l|}{$\begin{array}{l}\text { Total No. } \\
\text { of Cases }\end{array}$} & $\begin{array}{l}\text { CVS } \\
\text { Symptoms } \\
\text { Present }\end{array}$ & \multicolumn{2}{l|}{$\begin{array}{l}\text { No } \\
\text { Symptoms }\end{array}$} \\
\hline Total & $\%$ & Total & $\%$ & Total & $\%$ \\
\hline $\begin{array}{l}\text { Score >> } \\
3\end{array}$ & 15 & 10 & 15 & 100 & 0 & 0 \\
\hline $\begin{array}{l}\text { Score << } \\
3\end{array}$ & 24 & 16 & 19 & 79.16 & 5 & 20.84 \\
\hline Score 0 & 111 & 74 & 80 & 72.1 & 31 & 27.9 \\
\hline Total & 150 & 100 & 114 & 76 & 36 & 24 \\
\hline
\end{tabular}

Table 7: Comparision of Schirmer's Type-2 Test

\begin{tabular}{|l|l|l|l|l|l|l|}
\hline \multirow{2}{*}{ Variables } & \multicolumn{2}{|l|}{$\begin{array}{l}\text { Total No. of } \\
\text { Cases }\end{array}$} & $\begin{array}{l}\text { CVS } \\
\text { Symptoms } \\
\text { Present }\end{array}$ & \multicolumn{2}{|c|}{$\begin{array}{l}\text { No } \\
\text { Symptoms }\end{array}$} \\
\cline { 2 - 7 } & Total & $\%$ & Total & $\%$ & Total & $\%$ \\
\hline $\begin{array}{l}\text { TBUT } \\
(<10 s e c)\end{array}$ & 56 & 37.4 & 49 & 88 & 7 & 12 \\
\hline $\begin{array}{l}\text { TBUT } \\
(>10 s e c)\end{array}$ & 94 & 62.6 & 65 & 69 & 29 & 31 \\
\hline Total & 150 & 100 & 114 & 76 & 36 & 24 \\
\hline
\end{tabular}

Table 8:- Distribution of Fluroscein Staining In Subjects

\begin{tabular}{|l|l|l|l|l|l|l|}
\hline $\begin{array}{l}\text { Fluroscein } \\
\text { Staining }\end{array}$ & \multicolumn{2}{|l|}{ Total } & \multicolumn{2}{l|}{$\begin{array}{l}\text { Cvs } \\
\text { Symptoms } \\
\text { Present }\end{array}$} & \multicolumn{2}{l|}{$\begin{array}{l}\text { No } \\
\text { Symptoms }\end{array}$} \\
\hline All 5 quadrants & Total & $\%$ & Total & $\%$ & Total & $\%$ \\
\hline $\begin{array}{l}\text { Score More } \\
\text { Than 3 }\end{array}$ & 15 & 10 & 15 & 100 & 0 & 0 \\
\hline $\begin{array}{l}\text { Score Less } \\
\text { Than 3 }\end{array}$ & 24 & 16 & 19 & 79.16 & 5 & 20.84 \\
\hline Score 0 & 111 & 74 & 80 & 72.1 & 31 & 27.9 \\
\hline Total & 150 & 100 & 114 & 76 & 36 & 24 \\
\hline
\end{tabular}

Table No.9- Distribution of Lissamine Green Staining in Subjects among Study Participants

Table 10 - distribution of cases according to conjunctival impression cytology findings in computer users

\begin{tabular}{|l|l|l|l|l|l|l|}
\hline \multirow{2}{*}{$\begin{array}{l}\text { Conjunctival } \\
\text { Impression }\end{array}$} & \multicolumn{2}{|l|}{ Total } \\
$\begin{array}{l}\text { Cytology } \\
\text { Grading }\end{array}$ & Total & $\%$ & \multicolumn{2}{|l|}{$\begin{array}{l}\text { CVS } \\
\text { Symptoms }\end{array}$} & \multicolumn{2}{l|}{$\begin{array}{l}\text { No } \\
\text { Pymptoms }\end{array}$} \\
\cline { 2 - 7 } & 79 & 52.66 & 69 & 87.34 & 13 & 16.45 \\
\hline $\begin{array}{l}\text { Normal } \\
\text { Cytology }\end{array}$ & 25 & 16.66 & 22 & 88 & 3 & 12 \\
\hline $\begin{array}{l}\text { Mild } \\
\text { Dysplasia }\end{array}$ & 7 & 4.66 & 5 & 71.42 & 2 & 28.57 \\
\hline $\begin{array}{l}\text { Moderate } \\
\text { Dysplasia }\end{array}$ & 3 & 2 & 3 & 100 & 0 & 0 \\
\hline $\begin{array}{l}\text { Severe } \\
\text { Dysplasia }\end{array}$ & 31 & 20.66 & 15 & 48.38 & 16 & 51.61 \\
\hline Acelluar & 5 & 3.33 & 5 & 100 & 0 & 0 \\
\hline $\begin{array}{l}\text { Acute } \\
\text { Inflammatory } \\
\text { Lesions }\end{array}$ & 150 & 100 & 111 & 74 & 39 & 26 \\
\hline Total & & & & & & \\
\hline
\end{tabular}

\section{DISCUSSIONS}

This study included 150 cases of computer users examined at MYH. The prevalence of CVS was $75.33 \%$. Different outcome were reported on comparing the results with other studies AK Sharma et al (2006) ${ }^{[5]}$ reported prevalence of $76 \%$, Zairina et al $(2011)^{[6]}-68.1 \%$, Seshadhri et al $(2014)^{[7]}$ $69.3 \%$

In our study knowledge about Computer vision syndrome was present in 65 participants $(43.33 \%)$. Other studies Akinbinu et al (2013) ${ }^{[8]}$ reported $27 \%$ and Huda Zainuddin et al (2014) ${ }^{[9]}$ reported $35.6 \%$ and Amirul F Z (2015) ${ }^{[10]}$ reported $48.8 \%$ participants had knowledge about CVS.

The most experienced symptoms were Headache$101(67.33 \%$,$) , tired eyes 76(50.66 \%)$ and pain behind eyes 74 (49.33\%). The duration of computer work is directly related to eye symptoms and longer 
duration tends to result in long lasting complaints even after work is finished. Stella C et al (2007) ${ }^{[10]}$ Eyestrain (42.7\%), blurred vision (45.7\%) and Saurabh Shrivastava et al (2012) [11]_ Redness (40.2\%), stiffness in neck (45.2\%) Kp Mashige et al (2013) ${ }^{[13]}$ - Eyestrain (89\%), headache $81 \%$.

In our study, majority $64.66 \%$ of study participants were using computer daily for 4-6 hours.

Other studies like Stella C et al (2007) ${ }^{[10]}$, Zairinia Et al (2011) ${ }^{[6]}$ and Lograj et al (2014) ${ }^{[12]}$ also reported significant association of long working hours with computer and adverse effect.

Following strategies have been reported by researchers to reduce the symptom of CVS- Taking regular breaks in between use of computer was the most preventive measure taken for relief of symptoms in form of rule 20/20/20 as suggested by Anshel (2005) ${ }^{[14]}$ i,e after 20 minutes of computer use , one should look at distant object 20 feet away for 20 seconds, maintaining good sitting posture, use of eyedrops, use of antiglare screen has been reported to reduce the symptoms of CVS.

In the current study dry eye workup findings showed abnormal TBUT - 37.4\% \& positive schirmer's test- $28 \%$ of study participants, fluroscein staining findings reported in $10 \%$ and lissamine green staining findings were reported in $13.33 \%$.

Conjunctival impression cytology showed normal cytology in $53.33 \%$, mild dysplasia in $16.66 \%$, moderate dysplasia in $4.6 \%$ and severe dysplasia in $2 \%$ of study participants. Other studies Alireza Dehghani et al (2008) ${ }^{[15]}$ reported positive schirmer's findings in $38.5 \%$, Sanjeev kumar et al (2013) [16] reported conjunctival impression cytology- stage 4 in $90 \%$ and Rahul Bhargava et al (2014) ${ }^{[17]}$ reported TBUT- $48.5 \%$, Schirmer's test$38.4 \%$ and Conjunctival impression cytology abnormal in $38.4 \%$

\section{CONCLUSION}

In conclusion, in our study males were predominantly affected because of their nature of work. The age group of 26-30 years formed the core group of people to get symptoms of CVS.
Though, in our study the sample size was small the final outcome showed majority of computer users were suffering from Computer Vision Syndrome. Proper knowledge, work station setup and regular follow up with ophthalmologist and physician can reduce the computer related morbidity and improve the work performance among computer users.

\section{REFERENCES}

1. Loh KY, Reddy SC. Understanding and preventing computer vision syndrome. Malaysian Family Physician. 2008; 3 (3): 128-30

2. Tamez, G.S., Ortiz, H.L., Martinez, A.S. and Mendez, R., Salud. Publica. Mex., 45, 171-180 (2003)

3. American Optometric Association (2010). The effects of video display terminal use on eye health and vision. Available at:http://www.aoa.org/optometrists/education-and-training/clinicalcare/effects-of-video-display?

4. Blehm C, Vishnu S, Khattak A, Mitra S, Yee RW. Computer vision syndrome: A review. Surv Ophthalmol 2005;50:25362

5. AK Sharma, S Khera, J Khandekar. Computer realted health problems among IT professionals in Delhi. CME: 2006/ vol: 31/ issue : 1/ 36-38.

6. Zairina; Computer User:Demographic and Computer Related Factors that p.redispose user to get Computer Vision Syndrome. International Journal of Business, Humanities and Technology Vol. 1 No.2; September 2011

7. Seshadhri; Prevalence of Computer Vision Syndrome among Information Technology Professionals Working in Chennai. World Journal of Medical Sciences 11 (3): 312-314. 2014

8. T.R.Akinbinu (2013) Computer vision syndrome: a study of knowledge and practices in university students. Nepal $\mathbf{J}$ Ophthalmol 2013:5 (10): 161-168 
9. Huda Zainuddin, Knowledge of computer vision syndrome among computer users in the workplace in AUJA, Nigeria. Journal of Physiology and Pathophysiology Vol. 4(4),pp. 58-63 september, 2013

10. Stella C, Chiemeke, Allen E. Evaluation of Vision-realted problems amongst computer users: a case study of university of Benin, Nigeria. Proceedings of the World Congress of Engineering 2007 Vol I WCE 2007, July 2-4, 2007, London, U.K.

11. Saurabh R Shrivastava; Computer related health problems among software professionals in Mumbai: A crosssectional study. International Journal of Health \& Allied Sciences. Vol.1.Issue 2.Apr-Jun 2012

12. Lograj M; Computer Vision Syndrome and Associated Factors Among Medical and Engineering Students in Cheenai. Annals of Medical and Health Sciences Research / Mar-Apr 2014/ Vol 4 /Issue 2

13. KP Mashige, N Rampersad, OA Oduntn. A study of ergonomic factors leading to computer vision syndrome among computer users. Ergonomics SA, 2013,25(1) ISSN no.1010-2728.

14. Anshel J. Visual Ergonomics Handbook. In: Anshel J, editor. New York: Taylor and Francis; 2005

15. Alireza dehghani; Prevalence of ocular symptoms and signs among professional computer users in ISFAHAN, IRAN

16. Sanjeev kumar (2013) Conjunctival impression cytology in computer users. Nepal J Ophthalmol 2013:5(9): 33-37

17. Diagnostic value and accuracy of conjunctival impression cytology, dry eye symptomatology and routine function tests in computer users. Journal of Laboratory Physicians / Jul-Dec 2014/ Vol-6/ Issue-2 